\title{
Cognitive behavioural group therapy for male perpetrators of intimate partner violence: a systematic review
}

Merete Berg Nesset ${ }^{1,2^{*}}$, Mariela Loreto Lara-Cabrera ${ }^{2,3,4}$, Therese Kristine Dalsb $\varnothing^{5}$, Sindre Andre Pedersen ${ }^{6}$, Johan Håkon Bjørngaard ${ }^{1,7}$ and Tom Palmstierna ${ }^{1,2,8}$

\begin{abstract}
Background: Violence against intimate partners is a worldwide public health problem. Cognitive behavioural therapy delivered in a group format is widely used for the treatment of men's violent behaviour towards their female partners. A Cochrane review about the effectiveness of this therapy from 2011 revealed a lack of controlled studies. Our aim is to update the current evidence on the effectiveness of cognitive behavioural group therapy on men's violent behaviour towards their female partner.
\end{abstract}

Methods: The Cochrane Library, the Campbell Collaboration Social, MEDLINE, PsychINFO, CINAHL, SCOPUS, Embase, Open Grey, Grey Literature Report, and Sociological Abstracts were searched for studies investigating the effectiveness of cognitive behavioural group therapy on intimate partner violence published in the period of January 1, 2010, to February 12, 2018. Manual searches were also performed to identify randomized and non-randomized controlled trials. Data extraction was done in duplicate. The primary outcome was the reduction in violent behaviour, and secondary outcomes were physical health, mental health, quality of life, emotion regulation, and substance use. Study quality was assessed with the Cochrane Collaboration's risk of bias tool and the Risk of Bias In Non-Randomized Studies of Interventions tool. A narrative summary was used to describe the review findings.

Results: We identified six new studies that met the inclusion criteria: four randomized controlled trials and two nonrandomized trials. Three of the randomized controlled trials found a reduction in intimate partner violence after treatment. The fourth randomized trial found that a subsample of responding partners reported a reduction in violence but no changes in the men's self-reported violence after treatment. No effect could be detected in the two nonrandomized studies. Analysis of risk of bias revealed mixed results, indicating both strengths and weaknesses.

Limitations: Only a limited amount of studies which scored as "low quality" were available.

Conclusions: There is still insufficient evidence to confirm that cognitive behavioural group therapy for perpetrators of intimate partner violence has a positive effect. Future research should focus on randomized controlled studies distinguishing between convicted and non-convicted populations where violent behaviour is the primary outcome.

Trial registration: CRD42016041493.

Keywords: Batterer, CBT, Cognitive therapy, Group therapy, Intimate partner violence, Mental health, Perpetrator, Randomized controlled trials, Systematic review

\footnotetext{
* Correspondence: Merete.b.nesset@ntnu.no

${ }^{1}$ Forensic Department and Research Centre Brøset, St. Olav's University

Hospital, PO 1803 Lade, N-7440 Trondheim, Norway

${ }^{2}$ Faculty of medicine and health sciences, dept. of Mental Health, Norwegian

University of Science and Technology (NTNU), Trondheim, Norway

Full list of author information is available at the end of the article
}

(c) The Author(s). 2019 Open Access This article is distributed under the terms of the Creative Commons Attribution 4.0 International License (http://creativecommons.org/licenses/by/4.0/), which permits unrestricted use, distribution, and reproduction in any medium, provided you give appropriate credit to the original author(s) and the source, provide a link to the Creative Commons license, and indicate if changes were made. The Creative Commons Public Domain Dedication waiver (http://creativecommons.org/publicdomain/zero/1.0/) applies to the data made available in this article, unless otherwise stated. 


\section{Background}

Intimate partner violence is a violation of human dignity and rights and includes various forms of physical, sexual, and psychological abuse [1-4]. In contrast to other types of violent acts, violence by an intimate partner often reoccur within the relationship and can go on for years [3, 5], and recidivism rates of $21 \%$ [6] to $42 \%$ [7] are reported. Violence against women is a global public health problem and studies on intimate partner violence suggest that nearly one third of women experience physical or sexual violence from an intimate partner during their lifetime [8]. Furthermore, WHO [9] and others [10] estimated that as many as $38 \%$ of female homicides globally were committed by male partners, and the global life-time prevalence of physical and/or sexual violence by an intimate partner was $30 \%$. In addition, $20-75 \%$ of women have reported experiencing emotional violence [11].

Cognitive Behavioural Therapy (CBT) is one of the most actively researched psychotherapies and has received consistent empirical support for a host of mental health problems and conditions $[12,13]$. In the treatment of aggressive behaviour, CBT interventions are now a commonly used approach to help different populations to regulate anger and aggressive behaviour [14]. The main techniques used in CBT focus on establishing a therapeutic relationship, behavioural change strategies, cognitive restructuring, modification of core beliefs and sche mas, and the prevention of relapse and recurrence. Cognitive theory suggests that psychopathology is characterized by the activation of a conglomerate of related or contiguous dysfunctional beliefs, meanings, and memories that operate in coordination with affect, motivation, behaviour, and physiological responses [12]. Different psychopathological conditions are associated with specific biases that influence how an individual incorporates and responds to new information $[12,13]$.

CBT is commonly used to address dysfunctional anger and violent behaviour among intimate partners. Research on the effectiveness of such interventions has yielded mixed results $[15,16]$. A systematic review in 2007 identified six studies $(N=2343)$ which consisted of a mix of convicted and non-convicted male participants [16]. One study $(N=218)$ compared feminist-cognitive-behavioural-group-therapy with process-psychodynamic group therapy [17]. The second study $(N=64)$ compared a 12 -week CBT-based substance abuse and domestic violence group with a 12-week twelve-step facilitation group [18]. The results were inconclusive in each of the two studies. The other four studies compared CBT with no intervention (1771 participants in total) [19-22]. Only one of these showed a statistically significant effect in favour of CBT [22]. A meta-analysis showed that the relative risk for violence was 0.86 in favour of the intervention group with a confidence interval of $0.54-1.38$. However, a combination of a low effect size and a wide confidence interval led to the conclusion that there was insufficient evidence concerning the effectiveness of CBT. A revision of this study in 2011 failed to identify new randomized controlled trials, precluding any new meta-analyses [23].

The primary aim of this systematic review is to examine new evidence for the effectiveness of group-based CBT on men's violent behaviour towards their female partners. Secondly, we also review whether cognitive behavioural group therapy (CBGT) affects changes in self-reported physical health, mental health, quality of life, emotional regulation, substance use, and socioeconomic outcome among perpetrators.

\section{Methods}

The systematic review was registered in the International Prospective Register of Systematic Reviews (PROSPERO), no: PROSPERO 2016:CRD42016041493, and conducted according to the Preferred Reporting Items for Systematic Reviews and Meta-Analyses (PRISMA) standards [24, 25].

\section{Eligibility criteria}

1) Adult male participants aged 18 years or older who had a history of physical, psychological, or sexual violence towards their female intimate partners.

2) Participants voluntarily referred or convicted to treatment.

3) Studies examining the effect of cognitive behavioural group therapy.

4) The control group condition should be classified as applying no intervention, another intervention, or a waiting list.

5) The study should report on type, frequency and recurrence of physically, psychologically and/or sexually violent behaviour.

6) Eligible studies were required to be randomized or non-randomized controlled studies published in peer-reviewed journals during the publication period of January 1, 2010, to February 12, 2018.

7) The studies were written in English, Spanish, or Portuguese.

8) Studies examining perpetrators of human trafficking, child exposure to intimate partner violence, or dating violence among adolescents were excluded. Also, studies examining other forms of therapy than cognitive behavioural group therapy (i.e. couple's therapy, individual therapy) were excluded.

\section{Search strategy}

A systematic literature search was conducted with the assistance of a medical research librarian (S.A.P) on 
various databases: the Cochrane Library, the Campbell Collaboration Social, MEDLINE, PsychINFO, CINAHL, SCOPUS, Embase, Open Grey, Grey Literature Report, and Sociological Abstracts. The queries involved a combination of thesaurus and free-text terms that were optimised to identify studies on intimate partner violence and cognitive therapy in the respective databases (see additional file 1), building on a search strategy described by Smedslund et al. [16]. The search was limited to the period of January 1, 2010, to February 12, 2018, in order to find studies published since the review by Smedslund et al. [23]. In addition to examining the reference lists of included studies, the Journal of Interpersonal Violence and Journal of Family Violence were searched by hand for the relevant period.

\section{Data extraction}

Two authors (M.B.N and M.L.L-C) independently scree ned the abstracts and titles of the retrieved references and assessed the full text of potentially eligible studies. Discrepancies were resolved by discussion with a third author (T.P). Two authors (M.B.N and M.L.L-C) extracted data from all included articles by following the Template for Intervention Description and Replication (TIDIeR) [26]. The items extracted and recorded were the study design, setting, sample characteristics like age, voluntarily or court-ordered to treatment, as well as outcomes, treatment fidelity and length of follow-up. Moreover, type of intervention, type of control condition, measurement tools, and timing of the outcome assessment. The predefined secondary outcomes were also recorded. We contacted authors for further information if needed. The final decisions on which studies that met the inclusion criteria were made after discussion among the review authors.

\section{Quality assessment}

To determine the validity of randomized trials, three authors (M.B.N, M.L.L-C \& T.D) worked independently using the Cochrane Collaboration's risk of bias tool [27]. The same authors assessed the remaining studies using the Risk of Bias In Non-randomized Studies of Interventions (ROBINS-I) tool [28]. This process was followed by a discussion between all authors about the methodological quality of the included studies.

\section{Results}

\section{Search results}

The database searches yielded 4570 unique references (see Fig. 1, study flow diagram depicted from RevMan) [29]. Hand searching of the bibliographies of the systematic reviews and articles selected for the full text review revealed one additional study with potential relevance [30]. The full text of 16 articles was retrieved and reviewed in detail. One of these studies was excluded because it investigated the effect of individual therapy [31], while another was excluded because it investigated couples' therapy [32]. One was excluded because it did not measure violent behaviour but rather thoughts and aggressive feelings [33], and four additional studies were excluded because the main intervention was not group CBT [30, 34-36].

\section{Characteristics of included studies}

A total of six studies were finally included in the study following the screening process. Table 1 presents the characteristics of the four randomized controlled trials with 731 participants [37-40]. Table 2 presents the characteristics of the two non-randomized studies with 854 participants, one was a controlled retrospective cohort study [41], while the other was a quasi-experimental study [42]. The studies were conducted in Norway [39], United States [37, 38, 40], Sweden [41], and Spain [42] and published in English except for the study by Boira et al. [42], which was published in Spanish. The interventions described were carried out within special health services, a community setting serving victims and perpetrators of domestic violence, a prison or probation service setting, and a university setting.

The participants were recruited voluntarily or court-referred for treatment. Most of the participants in the studies were convicted of intimate partner violence. However, there were large notable differences concerning participant samples between the studies, ranging from 26 to 528 in the randomized controlled studies and between 62 and 792 in the non-randomized studies. The mean age of participants ranged from 34 to 40 years old.

The interventions used in the studies varied in content, length and how they were delivered. Palmstierna et al. [39] investigated the effect of cognitive behavioural group therapy (CBGT) delivered in a combination of three to four individual sessions followed by 15 two-hour group sessions. Alexander et al. [37] investigated the effect of 26 sessions of standard CBGT gender re-education. Murphy et al. [38] investigated the effect of 20 weekly 2-h sessions CBGT. Taft et al. [40] investigated the effect of 12 weekly 2 -h sessions of trauma informed group intervention.

With regard to the non-randomised studies Haggård et al. [41] investigated the effect of an integrated domestic abuse program (IDAP) consisting of a minimum of 8 individual sessions and 27 two-hour group sessions, while Boira et al. [42] investigated the effect of a 20-session manualised CBGT-program.

In all studies, the group leaders were therapists trained on intervention with perpetrators of intimate partner violence (psychologists, doctoral students in clinical psychology, clinical psychology graduate student trainee, 


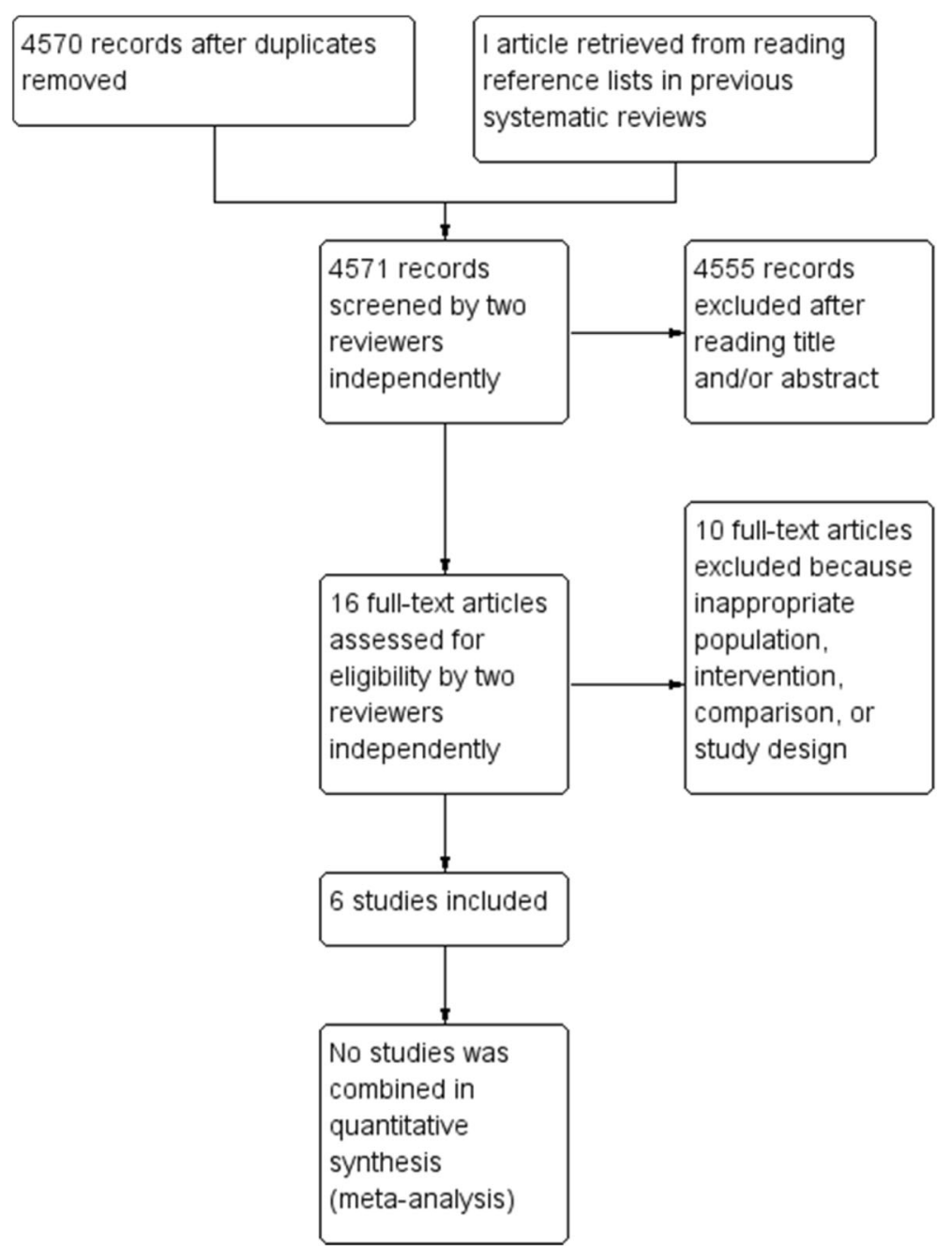

Fig. 1 Flow diagram depicting the stages in the study selection process

social workers, mental health nurses, or others with a university degree in behavioural science). The control groups were based on usual care [40, 41], an alternative intervention [37], a waiting list [39], or a comparison of the intervention with an open group format, individual therapy, or a waiting list [42], 20 sessions of standard individual cognitive therapy [38]. The intervention fidelity was measured in one study [37] by a blinded rater who listened to randomly selected audiotapes. Two studies reported treatment fidelity by recording group sessions followed by supervision to the instructors [38, 41].

\section{Quality assessment}

The risk-of-bias ratings for the randomized controlled trials are displayed in Fig. 2 a and b, depicted from RevMan [29]. All the included randomized trials in this review are judged as having poor quality. Additional file 2 shows more detailed information about the risk of bias ratings of each study.
Alexander et al. [37], Murphy et al. [38], Palmstierna et al. [39] and Taft et al. [40], score a high overall risk of bias in reporting according to the recommendations in the Cochrane Collaboration's tool for assessing risk of bias in randomised trials [27]. Alexander et al. [37] provide unclear information about the random sequence generation process, while the other three randomized controlled trials score a low risk of bias due to a detailed description of the random sequence generation. In all the four studies, the allocation concealment scores indicate that the risk is unclear due to inadequate description. Neither the participants nor the personnel were blinded to the treatment conditions in the four studies and therefore scored as high risk, although the research assistants making follow-up phone calls to the partners were blinded to the condition in the study by Alexander et al. [37].

With regard to incomplete data, two of the studies present no intention-to-treat analyses [37, 39]. While 


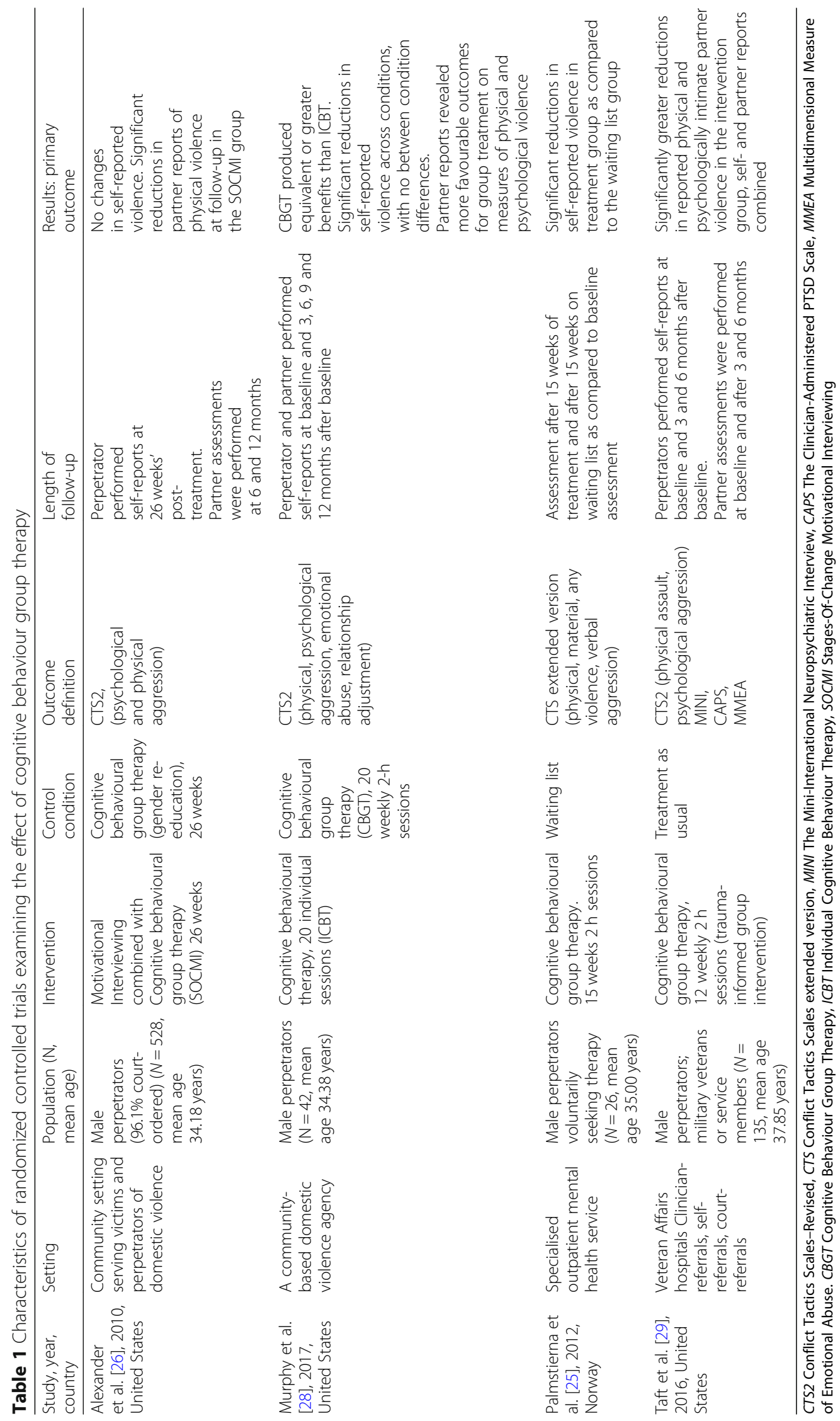


Table 2 Characteristics of non-randomized studies examining the effect of cognitive behavior group therapy

\begin{tabular}{|c|c|c|c|c|c|c|c|}
\hline $\begin{array}{l}\text { Study, } \\
\text { year, } \\
\text { country }\end{array}$ & Setting & $\begin{array}{l}\text { Population } \\
\text { (N, mean } \\
\text { age) }\end{array}$ & Intervention & $\begin{array}{l}\text { Control } \\
\text { condition }\end{array}$ & $\begin{array}{l}\text { Outcome } \\
\text { definition }\end{array}$ & $\begin{array}{l}\text { Length of follow- } \\
\text { up }\end{array}$ & $\begin{array}{l}\text { Results: } \\
\text { primary } \\
\text { outcome }\end{array}$ \\
\hline $\begin{array}{l}\text { Haggård } \\
\text { et al. [31], } \\
\text { 2017, } \\
\text { Sweden }\end{array}$ & $\begin{array}{l}\text { Prison and } \\
\text { probation } \\
\text { offices }\end{array}$ & $\begin{array}{l}\text { Consecutive } \\
\text { sample of } \\
\text { male IPV } \\
\text { perpetrators: } \\
\text { ( } N=792, \\
\text { mean age } \\
39.55 \text { years) }\end{array}$ & $\begin{array}{l}\text { Manual-based } \\
\text { group program } \\
\text { for male } \\
\text { perpetrators } \\
\text { (IDAP), including } \\
\text { a pro-feminist } \\
\text { psychoedu } \\
\text { cational approach }\end{array}$ & $\begin{array}{l}\text { Concomitant } \\
\text { IPV offender } \\
\text { controls } \\
\text { who did not } \\
\text { enter IDAP }\end{array}$ & $\begin{array}{l}\text { Any new } \\
\text { convictions } \\
\text { for any } \\
\text { violent } \\
\text { recidivism } \\
\text { and IPV } \\
\text { during the } \\
\text { follow up time }\end{array}$ & $\begin{array}{l}\text { From time } \\
\text { of recruitment } \\
\text { unto study } \\
\text { (2004-2007) } \\
\text { until March 2, } \\
2011 . \text { Mean } \\
\text { time at } \\
\text { risk, 4.6 years }\end{array}$ & $\begin{array}{l}19 \%(N=65) \\
\text { of IDAP } \\
\text { participants } \\
\text { and 19\% } \\
(N=84) \\
\text { controls } \\
\text { recidivated } \\
\text { in violence } \\
\text { against a } \\
\text { partner or } \\
\text { former } \\
\text { partner }\end{array}$ \\
\hline $\begin{array}{l}\text { Boira et a. } \\
\text { [32], 2013, } \\
\text { Spain }\end{array}$ & $\begin{array}{l}\text { Setting unclear. } \\
\text { Treatment delivered by } \\
\text { psychologists specialized in } \\
\text { intimate partner } \\
\text { violence }\end{array}$ & $\begin{array}{l}\text { Male } \\
\text { perpetrators } \\
\text { convicted for } \\
\text { IPV and court } \\
\text { ordered to } \\
\text { treatment } \\
\text { ( } N=62 \text {, } \\
\text { mean age } \\
39.70 \text { years) }\end{array}$ & $\begin{array}{l}\text { Three treatment } \\
\text { modalities: } \\
\text { 1. Structured group } \\
\text { 2. Unstructured } \\
\text { group (open } \\
\text { group format) } \\
\text { 3. Individual therapy }\end{array}$ & Waiting list & $\begin{array}{l}\text { Police reports } \\
\text { on new } \\
\text { intimate } \\
\text { partner } \\
\text { violence }\end{array}$ & 18 months & $\begin{array}{l}6.4 \% \text { of the } \\
\text { participants } \\
\text { across the } \\
\text { interventions } \\
\text { were reported } \\
\text { to the police } \\
\text { for new intimate } \\
\text { partner violence }\end{array}$ \\
\hline
\end{tabular}

CBT Cognitive Behavioural Therapy, IDAP Integrated Domestic Abuse Program, IPV Intimate Partner Violence

Murphy et al. [38], Palmstierna et al. [39] and Taft et al. [40] describe the distribution of attrition across groups, Alexander et al. [37] do not (they refer to another publication based on the same study). All four studies score as having high risk of bias for this item.

The risk of bias due to selective reporting is mixed across the studies. We found no protocol information on ClinicalTrials.gov for either Palmstierna et al. [39] or Alexander et al. [37]. Alexander et al. [37] only report on subjects completing the intervention and score at unclear risk of bias. Regarding other bias in the study of Alexander et al. [37], there is no power calculation or description of the how data were analysed and we suspect low statistical power. Palmstierna et al. [39] presents the results from self-reports of outcomes using the CTS and the associated $p$-values. However, the study only reports per-protocol results and gives no estimates of differences in reduced violence between the groups, hence this study is at high risk of bias on this domain. Taft et al. [40] did not report on the pre-defined secondary outcomes as stated in the Clinical Trials register, and score at high risk of bias on this domain. Murphy et al. [38] report all expected primary outcomes in the pre-specified way stated in the Clinical Trials register, and hence score at low risk of bias. With regard to other bias in the study of Murphy et al. [38], the imbalanced lack of compliance with allocated interventions between groups could cause bias and low statistical power. Hence, the study is at high risk of bias on this domain.

With respect to other sources of bias, Palmstierna et al. [39] were funded through the authors' employment at St. Olav's University Hospital and the Norwegian
University of Science and Technology. Alexander et al. [37] were supported by the National Institute of Justice Grant. Murphy et al. [38] was funded by a grant from the National Institutes of Health, and Taft et al. [40] was supported by grants from the Department of Veterans Affairs and Department of Defence and through the use of the facilities and resources of the Providence Veterans Affairs Medical Center.

One of the non-randomized studies [41] is judged as having an overall moderate risk of bias, while the second study as having an overall serious risk of bias [42] (Table 3) according to ROBINS-I [28]. With regard to bias due to confounding, the study by Haggård et al. [41] statistically controlled for baseline recidivism risk that might have confounded the association between treatment status and recidivism in violent behaviour. The study by Boira et al. [42] scores as having low risk of bias due to confounding since the participants were selected from the target population and the study controlled for possible baseline confounding. Furthermore, the four groups had comparable sociodemographic characteristics.

Both studies are judged as having low risk of bias in the selection of participants for the study since both include all participants eligible for the target trial. Both studies clearly define the intervention and control groups and score as low risk on bias in the classification of interventions. Haggård et al. [41] followed an intention-to-treat approach and are therefore judged as having low risk of bias due to deviations from intended interventions. Boira et al. [42] provided insufficient information about intention-to-treat analysis, adherence to the interventions 


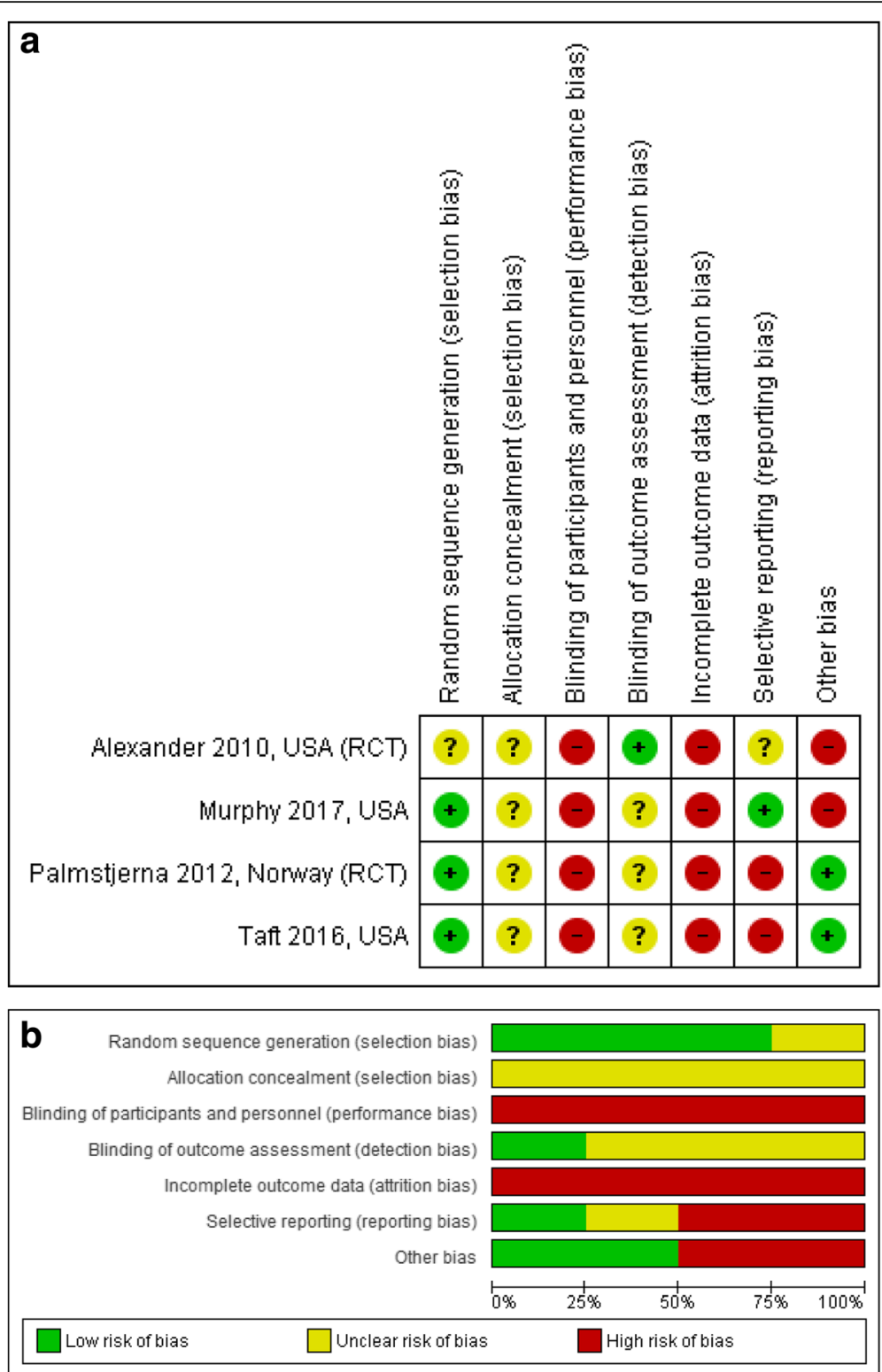

Fig. 2 a Risk of bias summary: review authors' judgements about each risk of bias item for each included study. b Risk of bias graph: review authors' judgements about each risk of bias item for each included study

and about the control group outcomes at post-test, hence there is no information to judge this item.

Risk of bias due to missing data is judged as low for Haggård et al. [41] since the study is retrospective and based on register data. Hence, no attrition from the study would affect the outcome. Furthermore, the study provides complete outcome measurements based on registry information. In the study of Boira et al. [42] there is insufficient information to judge this item. They report low attrition from the study but provide unclear information on missing data besides that.

The retrospective study by Haggård et al. [41] is judged as having low risk of bias in the measurement of outcomes since the results were already reported and the methods of outcome assessment were comparable across the intervention and control groups. Moreover, one assessor was blinded to recidivism data on any crime conviction in the past 5 years, any previous conviction of IPV, any previous conviction of a sexual offense, young age (below 21) at first known crime, any previous conviction of violation of a restraining order, current abuse or dependence on alcohol or drugs. The study by Boira et al. [42] is judged as having moderate risk of bias on this item due to a lack of blind outcome assessments and unclear information on the outcomes and intervention status for 18 months of follow up (the 


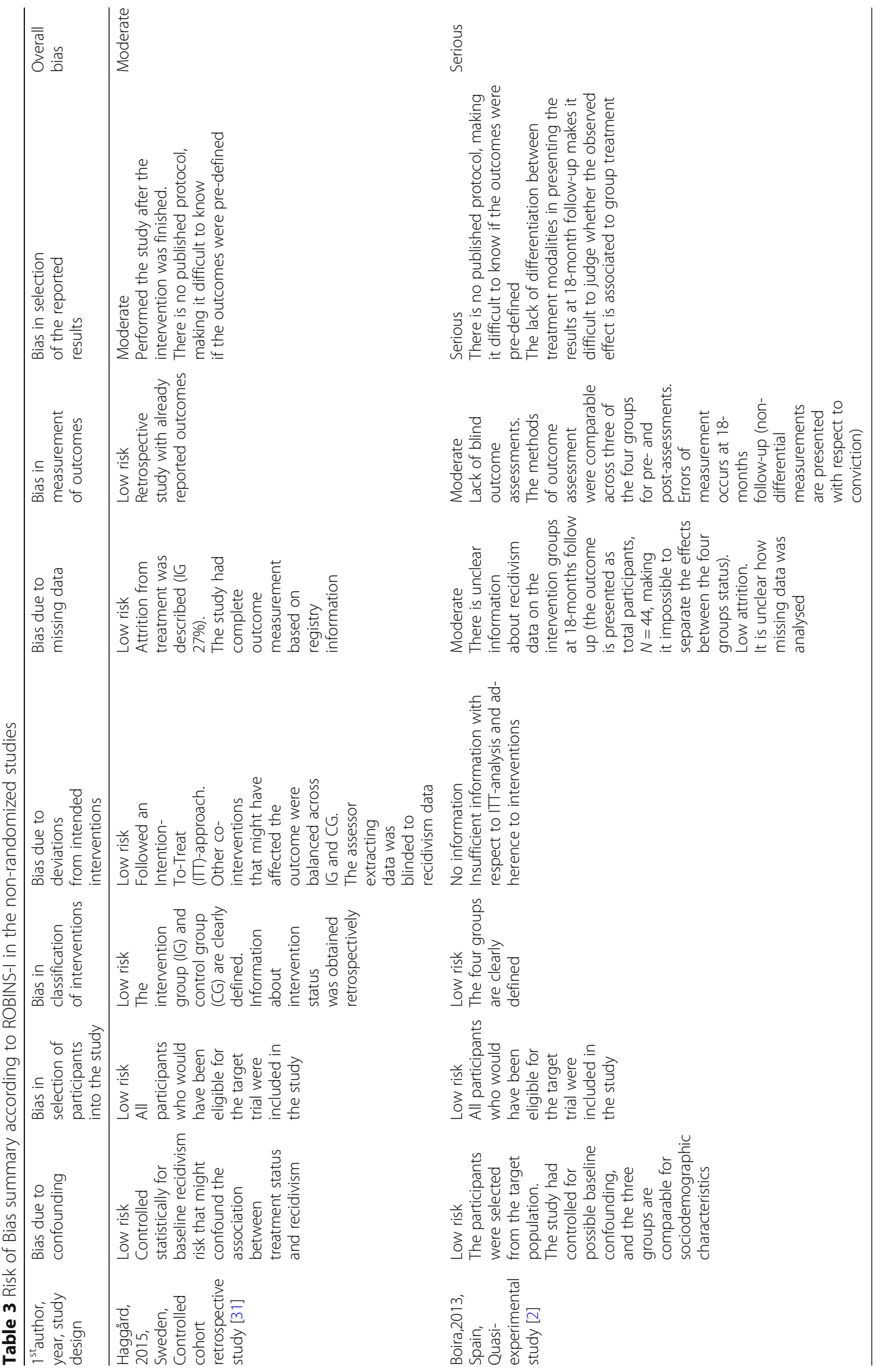


outcome is presented as the total participants $(N=44)$, making it impossible to separate the effects between the status of the four groups).

No published protocol was found for either of the studies [41, 42], making it difficult to determine whether the outcomes were predefined. Also, in the Boira [42] study a lack of differentiation between treatment modalities in presenting the results at 18-month follow-up makes it difficult to judge whether the observed effect is associated with group treatment. The treatment programs used by Boira et al. [42] (personal communication) were not compared, and they instead measured the effect of each program separately on new reports of intimate partner violence.

With respect to other sources of bias, Haggård et al. [41] reported indirect funding from the Swedish Prison and Probation Service through the authors' employment there. Boira et al. [42] did not report funding but had a collaboration agreement between the General Secretariat of Penitentiary Institutions and the College of Psychologists of Aragon.

\section{Primary outcome: Effect on violent behaviour}

The reported primary and secondary outcomes are summarized narratively given the considerable diversity of how they were assessed and the report of data in the studies included. Tables 1 and 2 display the primary outcome measures. Four randomized controlled trials [37-40] including 731 clients and 202 partners, and two non-randomized studies $[41,42]$ including 854 clients report outcomes on violent behaviour. Four studies [37-40] assessed violent behaviour using the Conflict Tactics Scale (CTS/CTS2) [43]. One study obtained register data from the Swedish Prison and Probation Service and court records on reconviction for violent crime against an intimate partner [41], while another study used register data on intimate partner violence reported to the police [42]. Only one study addressed sexual violence [41].

The small study by Palmstierna et al. [39] $(N=26)$ indicates a protective effect of CBGT on self-reported violence related to intimate partners as compared to the waitlist control, immediately after the intervention. This study also finds a significant correlation between low age and continued physically violent behaviour. The substantially larger study by Alexander et al. [37] $(N=528)$ find no differences with respect to perpetrator self-reports of violence at the end of treatment between men assigned to a group treatment program based on the stages of change model and motivational interviewing (SOCMI) and those in a program based on the Duluth model-inspired CBT. Of the $43 \%$ of partners who responded, fewer partners in the SOCMI group reported having experienced physical aggression at follow-up. Murphy et al. [38] $(N=42)$ find that cognitive behaviour group therapy produces outcomes equal to or better than individual cognitive behaviour therapy. The difference between the two conditions are statistically significant for partner reports of psychological violence and exceed a medium effect size for physical assaults and emotional abuse. Taft et al. [40] $(N=135)$ report that the intervention was more effective than the control condition in reducing psychological and physical intimate partner violence, with a small-to-medium between-group effect size.

Haggård et al. [41] report that 19\% i.e. 65 of the 340 participants in the treatment group and $19 \%$ i.e. 84 of the 452 controls recidivated in violence against a partner or former partner during follow-up. In the small study by Boira et al. [42] ( $N=65$, four different comparison groups), the authors conclude that they cannot obtain any conclusive evidence on any of the many outcome measures. As for their primary outcome (police reports after 18 months on new intimate partner violence), they do not compare the three programs. Furthermore, they do not report differences between the programs and the control group, and $94 \%(N=44)$ of the program participants (regardless of study condition) did not have any new incidents of intimate partner violent reported to the police at 18-month follow-up. The primary outcome for the control group is not reported.

\section{Secondary outcomes}

None of the studies included report treatment effects on physical health, quality of life, emotional regulation, and substance use after treatment. Only Boira et al. [42] report the effects of treatment on mental health as measured by the Symptom Checklist-90 (SCL-90). They found lower scores after structured group therapy in the SCL-90 depression dimension, Global Severity Index, and total symptom load.

\section{Other measurements}

Two studies [37, 42] assess the participants' readiness to change using The University of Rhode Island Change Assessment (URICA) [44]. However, the use of URICA by Boira et al. [42] is not satisfactorily explained. Alexander et al. [37] report a different outcome on physical violence: those with high initial readiness to change benefit more from group CBT than those with low initial readiness to change.

One study [42] assess empathy using the Spanish version of the Interpersonal Reactivity Index [45, 46], as well as hostility measured by the Spanish version of the Buss-Durkee Hostility Inventory [47, 48]. One study [37] assess risk factors for repeated violence using 12 items of the Danger Assessment Scale (DAS) [49]. Two studies [38, 40] use The Multidimensional Measure of Emotional Abuse (MMEA) [50] as an additional measure to assess 
psychological intimate partner violence. One study [38] assess relationship adjustment using the Dyadic Adjustment Scale (DAS) [51], the participants' communication difficulties by partner reports on the Spouse Verbal Problem Checklist [52], and the participants' responses to challenging relationship scenarios using the Articulated Thoughts in Simulated Situations [53] paradigm.

\section{Discussion}

This systematic review evaluates and updates the evidence published on the effectiveness of cognitive behavioural group therapy for male perpetrators of intimate partner violence since the Cochrane review on this topic published in 2007 and replicated in 2011 [16, 23]. Only six studies met our inclusion criteria. Our main finding supports the results of the last updated review by Smedslund et al. [23] in 2011, that the evidence for this therapy is still inconclusive.

Three of the included studies found a reduction in physical violence among participants in the group-based interventions [38-40]. However, these studies were small and most of the findings relied solely on self-report from the perpetrators. The larger study by Alexander et al. [37] included 528 male participants and found only marginal differences in self-reported violence with respect to the type of treatment. Boira et al. [42] relied on police reports, which are known to capture only a small part of the actual incidents of intimate partner violence. Furthermore, the participants were not randomly selected for the different treatment modalities. Moreover, Boira et al. [42] had a wide range of outcomes without differentiation between primary and secondary outcomes. They reported only pre-post evaluations without comparing the group change differences. The study by Haggård et al. [41] reported the recurrence of intimate partner violence based on new convictions, which is also subject to the same limitation as the method by Boira et al. [42]: it does not necessarily show the true picture with regard to the violence that is actually occurring.

This review clearly confirms that self-reported outcomes like physical health, mental health, quality of life, emotional regulation and substance use are scarcely addressed when investigating the effectiveness of cognitive behavioural therapy for anger and aggressive behaviour. Future randomized controlled trials should therefore also address these outcomes.

A randomized controlled trial design is preferred when evaluating treatment effects due to confounding by indication. Nevertheless, only four of the six studies reviewed are randomized controlled trials. Furthermore, one of the studies has a limited sample size of only 26 participants [39].

When evaluating treatment effects, it is necessary to consider the treatment context [54, 55]. Delivering treatment within the prison service is different from an outpatient setting. The therapy is given in different settings across all the included studies, and most of the participants are involuntarily referred except for those examined in the study by Palmstierna et al. [39]. It is important to separate participants who are involuntarily assigned to treatment from those seeking treatment on their own initiative since they probably represent different subtypes of perpetrators with different associated risks of recurrent violence and treatment compliance [56, 57]. Earlier systematic reviews of cognitive therapy for perpetrators of intimate partner violence have not distinguished sufficiently between the different subtypes of perpetrators and the type of contexts where the treatment is delivered.

\section{Limitations and implications for future research}

Since only six studies met the inclusion criteria, the conclusions drawn from this review should be interpreted with caution. We found reasons to suspect that there is a high risk of bias across the included studies, mainly due to lack of blinding and incomplete outcome data reporting. Also, allocation concealment and other important domains were poorly reported and represents a threat to the certainty of the overall evidence.

Two of the studies used register data on convictions and police reports, and four studies used self- and/or partner reports. The lack of standardisation of the study design, follow-up time, and outcome measurement found in the included studies prevents us from performing a meaningful meta-analysis. Not reporting the outcomes according to the original random assignment violates the intention behind random assignment and makes the experiment less likely to take into account possible confounding by indication.

The scarce evidence on the effect of group-based CBT calls for well-conducted randomised controlled trials in different settings, as well as different and defined selections of perpetrators. The findings of this review underscore these important areas for future research, which is in line with earlier evidence on different treatment modalities for perpetrators of intimate partner violence [23, 58-60]. Our review and previous research on intimate partner violence programmes reveal that a combination of multiple theoretical models and treatment modalities are common in clinical practice, which makes outcome evaluations challenging $[55,61]$. In future research, the elements of the treatment should at least be described clearly to make it possible to evaluate and compare treatment effects [54]. It is also important to ascertain the therapeutic adherence to the protocol, which will increase the attribution of effects or lack of effects to the intervention. Furthermore, non-randomized studies should publish protocols including a pre-analysis plan. It 
is also recommended that randomized controlled trials use [26] and follow the CONSORT guidelines [62, 63].

\section{Conclusion}

The evidence is still inconclusive with regard to the effectiveness of group-based CBT in reducing violence from men towards their female partners - a situation that is due to a lack of high-quality randomized controlled trials on the subject. An important implication for future research in this area is to put an emphasis on describing the interventions in detail and reporting the study design and finally, how the study was carried out.

Our review also reveals that, so far, few studies have investigated how group-based CBT affects self-reported outcomes on physical health, mental health, quality of life, emotional regulation, substance use, and socioeconomic outcome among perpetrators. Based on our findings, future studies should adopt a randomized controlled study design with clear criteria for randomization, blinding and allocation concealment. Reduction of violent behaviour should be the primary outcome, as measured by both self-reports and partner-reports. A clear description of the investigated perpetrator population is warranted since studies of convicted perpetrators should be separated from studies of non-convicted.

\section{Additional files}

Additional file 1: Search strategies. A detailed description of the search strategies and the used search terms. (DOCX $19 \mathrm{~kb}$ )

Additional file 2: Risk of Bias Ratings. A detailed description of the risk of bias ratings of the included randomized controlled trials. (DOCX $20 \mathrm{~kb}$ )

\section{Abbreviations \\ CAPS: Clinician-Administered PTSD Scale; CBGT: Cognitive Behavioural Group Therapy; CBT: Cognitive Behavioural Therapy; CG: Control Group; CTS: Conflict Tactics Scale extended version; CTS2: Conflict Tactics Scales- Revised; DAS: Danger Assessment Scale; ICBT: Individual Cognitive Behavioural Therapy; IDAP: Integrated Domestic Abuse Program; IG: Intervention Group; IPV: Intimate Partner Violence; ITT: Intention-To-Treat; MINI: Mini-International Neuropsychiatric Interview; MMEA: Multidimensional Measure of Emotional Abuse; PRISMA: Preferred Reporting Items for Systematic Reviews and Meta-Analyses; PROSPERO: Prospective Register of Systematic Reviews; RCT: Randomized Controlled Trial; RevMan: Review Manager; RoB: Risk of Bias; ROBINS-I: Risk of Bias In Non-Randomized Studies of Interventions tool; SCL-90: Symptom Checklist-90; SOCMI: Stages Of Change model and Motivational Interviewing; TIDleR: Template for Intervention Description and Replication; URICA: University of Rhode Island Change Assessment}

\section{Acknowledgements}

We are grateful to Santiago Boira, $\mathrm{PhD}$, for helping to clarify information with regard to the results in their paper.

\section{Funding}

The review was funded by St. Olav's University Hospital, Forensic Department and Research Centre Brøset.

Availability of data and materials

Data and materials are available on request from the first author.

\section{Authors' contributions}

MBN and MLL-C provided the initial motivation for the systematic review, developed the protocol and participated in the data extraction process. MBN wrote the manuscript. SAP participated in the development of the protocol, conducted the searches and participated in the data extraction process. TD participated in the data extraction process and presided over differences between the authors as they arose during the evaluation of risk of bias. JHB and TP participated in the data extraction process and presided over differences between the authors as they arose during the data extraction process. All authors contributed to the development of the final version of the manuscript and gave approval to the final version to be published.

Ethics approval and consent to participate

Not applicable.

\section{Consent for publication}

Not applicable.

\section{Competing interests}

The authors declare that they have no competing interests in the conduct of this systematic review or the preparation of this manuscript.

\section{Publisher's Note}

Springer Nature remains neutral with regard to jurisdictional claims in published maps and institutional affiliations.

\section{Author details}

${ }^{1}$ Forensic Department and Research Centre Brøset, St. Olav's University Hospital, PO 1803 Lade, N-7440 Trondheim, Norway. ${ }^{2}$ Faculty of medicine and health sciences, dept. of Mental Health, Norwegian University of Science and Technology (NTNU), Trondheim, Norway. ${ }^{3}$ Tiller Community Mental Health Centre, Division of Psychiatry, St. Olav's University Hospital,

Trondheim, Norway. ${ }^{4}$ Department of Research and Development, Division of Mental Health, St Olav's University Hospital, Trondheim, Norway. ${ }^{5}$ Norwegian Institute of Public Health, Oslo, Norway. 'ibrary Section for Medicine and Health Sciences, NTNU University Library, NTNU - Norwegian University of Science and Technology, Trondheim, Norway. 'Department of Social Science, Norwegian University of Science and Technology (NTNU), Trondheim, Norway. ${ }^{8}$ Department of Clinical Neuroscience, Centre for Psychiatric Research, Karolinska Institutet, Stockholm, Sweden.

Received: 7 August 2018 Accepted: 1 January 2019

Published online: 08 January 2019

\section{References}

1. Devries K, Watts C, Yoshihama M, Kiss L, Schraiber LB, Deyessa N, Heise L, Durand J, Mbwambo J, Janssen $\mathrm{H}$, et al. Violence against women is strongly associated with suicide attempts: evidence from the WHO multi-country study on women's health and domestic violence against women. Soc Sci Med. 2011.

2. FRA European Union Agency for Fundamental Rights: Violence against women: an EU-wide survey. In.; 2014.

3. Garcia-Moreno C, Jansen HA, Ellsberg M, Heise L, Watts CH. Prevalence of intimate partner violence: findings from the WHO multi-country study on women's health and domestic violence. Lancet. 2006;368(9543):1260-9.

4. Heise L, Garcia-Moreno C. Violence by intimate partners. In: Krug LLD EG, Mercy JA, Zwi AB, Lozano R, editors. World report on violence and health edn. Geneva: World Health Organization; 2002. p. 87-121.

5. Vatnar SKB, Bjørkly S. Lethal intimate partner violence: an interactional perspective on women's perceptions of lethal incidents. Violence Vict. 2013; 28(5):772-89.

6. Belfrage H, Strand S, Storey JE, Gibas AL, Kropp PR, Hart SD. Assessment and management of risk for intimate partner violence by police officers using the spousal assault risk assessment guide. Law Hum Behav. 2011.

7. Belfrage $H$, Strand S. Measuring the outcome of structured spousal violence risk assessments using the B-SAFER: risk in relation to recidivism and intervention. Behavioral Sciences and the Law. 2012;30:420-30.

8. Devries KM, Mak JY, García-Moreno C, Petzold M, Child JC, Falder G, Lim S, Bacchus $L J$, Engell RE, Rosenfeld L. The global prevalence of intimate partner violence against women. Science. 2013;340(6140):1527-8. 
9. WHO, Department of Reproductive Health and Research, London School of Hygiene and Tropical Medicine, Council SAMR: Global and regional estimates of violence against women: Prevalence and health effects of intimate partner violence and non-partner sexual violence. In.; 2013.

10. Stöckl H, Devries K, Rotstein A, Abrahams N, Campbell J, Watts C, Moreno CG. The global prevalence of intimate partner homicide: a systematic review. Lancet. 2013;382(9895):859-65

11. García-Moreno C, Jansen HA, Ellsberg M, Heise L, Watts C: WHO multicountry study on women's health and domestic violence against women: initial results on prevalence, health outcomes and women's responses: World Health Organization; 2005.

12. Beck A, Dozois D. Cognitive therapy: current status and future directions. Ann Rev Med. 2011;62:397-409.

13. Epp AM, Dobson KS. The evidence base for cognitive-behavioral therapy. Handbook of cognitive-behavioral therapies. 2010:39-73.

14. Lee AH, DiGiuseppe RJ. Anger and aggression treatments: a review of metaanalyses. Curr Opin Psychol. 2018;19:65-74.

15. Henwood KS, Chou S, Browne KD. A systematic review and meta-analysis on the effectiveness of CBT informed anger management. Aggress Violent Behav. 2015;25:280-92.

16. Smedslund G, Dalsbo TK, Steiro AK, Winsvold A, Clench-Aas J. Cognitive behavioural therapy for men who physically abuse their female partner. Cochrane Database Syst Rev. 2007;3:CD006048.

17. Saunders DG. Feminist-cognitive-behavioral and process-psychodynamic treatments for men who batter: interaction of abuser traits and treatment models. Violence Vict. 1996;11(4):393-414

18. Easton CJ, Mandel D, Babuscio T, Rounsaville BJ, Carroll KM. Differences in treatment outcome between male alcohol dependent offenders of domestic violence with and without positive drug screens. Addict Behav. 2007;32(10):2151-63

19. Dunford FW. The San Diego navy experiment: an assessment of interventions for men who assault their wives. J Consult Clin Psychol. 2000;68(3):468-76.

20. Feder $L$, Dugan $L$. A test of the efficacy of court-mandated counseling for domestic violence offenders: the Broward experiment. Justice Q. 2002;19(2): 343-75.

21. Labriola M, Rempel M, Davis RC: Testing the effectiveness of batterer programs and judicial monitoring: results from a randomized trial at the Bronx misdemeanor domestic violence court: Center for Court Innovation new York; 2005.

22. Taylor BG, Davis RC, Maxwell CD. The effects of a group batterer treatment program: a randomized experiment in Brooklyn. Justice Q. 2001;18(1):171-201.

23. Smedslund G, Dalsbø TK, Steiro A, Winsvold A, Clench-Aas J. Cognitive behavioural therapy for men who physically abuse their female partner. Cochrane Database Syst Rev. 2011;2.

24. Moher D, Liberati A, Tetzlaff J, Altman DG, Group P. Preferred reporting items for systematic reviews and meta-analyses: the PRISMA statement. PLoS Med. 2009;6(7):e1000097.

25. Moher D, Shamseer L, Clarke M, Ghersi D, Liberati A, Petticrew M, Shekelle P, Stewart LA, Group P-P. Preferred reporting items for systematic review and meta-analysis protocols (PRISMA-P) 2015 statement. Systematic Reviews. 2015;4(1).

26. Hoffmann TC, Glasziou PP, Boutron I, Milne R, Perera R, Moher D, Altman DG, Barbour V, Macdonald H, Johnston M. Better reporting of interventions: template for intervention description and replication (TIDieR) checklist and guide. Bmj. 2014;348:g1687.

27. Higgins JP, Altman DG, Gotzsche PC, Juni P, Moher D, Oxman AD, Savovic J, Schulz KF, Weeks L, Sterne JA. The Cochrane Collaboration's tool for assessing risk of bias in randomised trials. Bmj. 2011;343:d5928.

28. Sterne JA, Hernan MA, Reeves BC, Savovic J, Berkman ND, Viswanathan M, Henry D, Altman DG, Ansari MT, Boutron I, et al. ROBINS-l: a tool for assessing risk of bias in non-randomised studies of interventions. Bmj. 2016; 355:14919.

29. The Nordic Cochrane Centre. Review manager (RevMan). version 5.3 ed. Copenhagen: The Cochrane Collaboration; 2014.

30. Quintas J, Fonseca E, Serra A, Sousa H: Programa para Agressores de Violência Doméstica: Avaliação do Impacto da Aplicação Experimental (2010-2011). In.; 2012.

31. Kraanen FL, Vedel E, Scholing A, Emmelkamp PM. The comparative effectiveness of integrated treatment for substance abuse and partner violence (I-StoP) and substance abuse treatment alone: a randomized controlled trial. BMC psychiatry. 2013;13:189.
32. Taft CT, Howard J, Monson CM, Walling SM, Resick PA, Murphy CM. "Strength at home" intervention to prevent conflict and violence in military couples: pilot findings. Partner Abuse. 2014;5(1):41-57.

33. Rodríguez-Espartal N, Lopez-Zafra E. Programa emocional para presos por violencia de género (PREMOVIGE): Efectividad en variables cognitivas y conductuales. Psychosoc Interv. 2013;22(2):115-23.

34. Cavanaugh MM, Solomon PL, Gelles RJ. The dialectical psychoeducational workshop (DPEW) for males at risk for intimate partner violence: a pilot randomized controlled trial. J Exp Criminol. 2011;7(3):275-91.

35. Cunha OS, Gonçalves RA. Efficacy assessment of an intervention program with batterers. Small Group Res. 2015;46(4):455-82

36. George DT, Phillips MJ, Lifshitz M, Lionetti TA, Spero DE, Ghassemzedeh N, Doty L, Umhau JC, Rawlings RR. Fluoxetine treatment of alcoholic perpetrators of domestic violence: a 12-week, double-blind, randomized, placebocontrolled intervention study. psychiatry J Clin Psychiatry. 2011;72(1):60.

37. Alexander PC, Morris E, Tracy A, Frye A. Stages of change and the group treatment of batterers: a randomized clinical trial. Violence Vict. 2010;25(5): $571-87$.

38. Murphy CM, Eckhardt $\mathrm{Cl}$, Clifford JM, Lamotte AD, Meis LA. Individual versus group cognitive-behavioral therapy for partner-violent men: a preliminary randomized trial. J Interpers Violence. 2017:886260517705666.

39. Palmstierna T, Haugan G, Jarwson S, Rasmussen K, Nøttestad JA. Cognitivebehaviour group therapy for men voluntary seeking help for intimate partner violence. Nord J Psychiatry. 2012;66(5):360-5.

40. Taft CT, Macdonald A, Creech SK, Monson CM, Murphy CM. A randomized controlled clinical trial of the strength at home Men's program for partner violence in military veterans. J Clin Psychiatry. 2016;77(9):1168-75.

41. Haggård U, Freij I, Danielsson M, Wenander D, Långström N. Effectiveness of the IDAP treatment program for male perpetrators of intimate partner violence: a controlled study of criminal recidivism. J Interpers Violence. 2017;32(7):1027-43.

42. Boira S, López Y, Tomás-Aragonés L, Gaspar AR. Efficacy of different treatment modalities in men convicted of inti-mate partner violence. Anales de Psicología/Annals of Psychology. 2013;29(1):19-28.

43. Straus MA, Hamby SL, Boney-McCoy S, Sugarman DB. The revised conflict tactics scales (CTS2) Development and Preliminary Psychometric Data. JFI. 1996;17(3):283-316.

44. McConnaughy EA, DiClemente CC, Prochaska JO, Velicer WF. Stages of change in PSYCHOTHERAPY: a follow-up report. Psychotherapy: Theory/ Research/Practice/Training Winter. 1989;26(4):494-503.

45. Davis MH. A multidimensional approach to individual differences in empathy. JSAS Catalog of Selected Documents in Psychology. 1980;10.

46. Pérez-Albéniz A, De Paúl J, Etxeberría J, Montes MP, Torres E. Adaptación de interpersonal reactivity index (IRI) al español. Psicothema. 2003;15(2):267-72.

47. Buss AH, Durkee A. An inventory for assessing different kinds of hostility. J Consult Psychol. 1957;21(4).

48. Montalván V, Graver R, Oquendo MA, Baca-García E, Morales M, Mann J. Spanish adaptation of the buss-Durkee hostility inventory (BDHI). Eur J Psychiatry. 2001;15(2):101-12.

49. Campbell JC, Webster DW, Glass N. The danger assessment. Validation of a lethality risk assessment instrument for intimate partner Femicide. J Interpers Violence. 2009;24(4):653-74.

50. Murphy CM, Hoover SA. Measuring emotional abuse in dating relationships as a multifactorial construct. Violence Vict. 1999;14(1):39-53.

51. Spanier GB. Measuring dyadic adjustment: new scales for assessing the quality of marriage and similar dyads. J Marriage Fam. 1976;38:15-28.

52. Haynes SN, Chavez RE, Samuel V. Assessment of marital communication and distress. Behavioral Assessment. 1984;6:315-21.

53. Zanov MV, Davison GC. A conceptual and empirical review of 25 years of cognitive assessment using the articulated thoughts in simulated situations (ATTS) think-aloud paradigm. Cogn Ther Res. 2010;34:282-91.

54. Craig P, Dieppe P, Macintyre S, Michie S, Nazareth I, Petticrew M. Developing and evaluating complex interventions: the new Medical Research Council guidance. Bmj. 2008:337:a1655.

55. Datta J, Petticrew M. Challenges to evaluating complex interventions: a content analysis of published papers. BMC Public Health. 2013;13(1):568.

56. Petersson J, Strand S, Selenius H. Risk factors for intimate partner violence: a comparison of antisocial and family-only perpetrators. J Interpers Violence. 2016;0886260516640547.

57. Thijssen J, de Ruiter $C$. Identifying subtypes of spousal assaulters using the B-SAFER. J Interpers Violence. 2011;26(7):1307-21. 
58. Akoensi TD, Koehler JA, Lösel F, Humphreys DK. Domestic violence perpetrator programs in Europe, part 2: a systematic review of the state of evidence. Int J Offender Ther Comp Criminol. 2012;57(10):1206-25.

59. Babcock J, Armenti N, Cannon C, Lauve-Moon K, Buttell F, Ferreira R, Cantos A, Hamel J, Kelly D, Jordan C, et al. Domestic violence perpetrator programs: a proposal for evidence-based standards in the United States. Partner Abuse. 2016;7(4):355-460.

60. Eckhardt Cl, Murphy CM, Whitaker DJ, Sprunger J, Dykstra R, Woodard K The effectiveness of intervention programs for perpetrators and victims of intimate partner violence. Partner abuse. 2013:4(2):196-231.

61. Hamilton L, Koehler JA, Lösel FA. Domestic violence perpetrator programs in Europe, part I: a survey of current practice. Int J Offender Ther Comp Criminol. 2013;57(10):1189-205.

62. Boutron I, Moher D, Altman DG, Schulz KF, Ravaud P. Extending the CONSORT statement to randomized trials of nonpharmacologic treatment: explanation and elaboration. Ann Intern Med. 2008;148(4):295-309.

63. Schulz KF, Altman DG, Moher D. CONSORT 2010 statement: updated guidelines for reporting parallel group randomised trials. BMC Med. 2010; 8(1):18.

Ready to submit your research? Choose BMC and benefit from:

- fast, convenient online submission

- thorough peer review by experienced researchers in your field

- rapid publication on acceptance

- support for research data, including large and complex data types

- gold Open Access which fosters wider collaboration and increased citations

- maximum visibility for your research: over $100 \mathrm{M}$ website views per year

At $\mathrm{BMC}$, research is always in progress.

Learn more biomedcentral.com/submissions 